\title{
Physical Activity Associates with Greater Antibody Persistence through 6 Months after the Second Dose of CoronaVac in Patients with Autoimmune Rheumatic Diseases
}

\author{
Bruno Gualano ( $\nabla$ gualano@usp.br) \\ University of Sao Paulo \\ Ítalo R. Lemes \\ University of Sao Paulo \\ Rafael Silva \\ University of Sao Paulo \\ Ana Jessica Pinto \\ University of Colorado \\ Bruna Mazzolani \\ University of Sao Paulo
}

Fabiana I. Smaira

University of Sao Paulo

Sofia M. Sieczkowska

University of Sao Paulo

Nadia Aikawa

University of Sao Paulo

Sandra Pasoto

University of Sao Paulo

Ana C. Medeiros-Ribeiro

University of Sao Paulo

Carla Saad

University of Sao Paulo

Emily Neves

University of Sao Paulo

Clovis Silva

University of Sao Paulo

Paul Swinton

Robert Gordon University

Pedro Hallal

Federal University of Pelotas 


\section{Hamilton Roschel}

University of Sao Paulo

\section{Eloisa Bonfa}

University of Sao Paulo

\section{Research Article}

Keywords: physical activity, vaccine responses, COVID-19, immunosuppression, autoimmune rheumatic disease

Posted Date: December 28th, 2021

DOI: https://doi.org/10.21203/rs.3.rs-1202511/v1

License: (c) (i) This work is licensed under a Creative Commons Attribution 4.0 International License.

Read Full License 


\section{Abstract}

This study aimed to investigate the association between physical activity and persistent anti-SARS-CoV-2 antibodies 6 months after two-dose schedule of CoronaVac in autoimmune rheumatic diseases (ARD) patients. This was a prospective cohort study within an open-label, single-arm, phase 4 vaccination trial (clinicaltrials.gov \#NCT04754698), conducted at a tertiary referral hospital in Sao Paulo, Brazil. ARD patients aged $\geq 18$ underwent a two-dose schedule of CoronaVac (Sinovac Life Sciences, China). Persistent immunogenicity 6 months after the full-course vaccination was assessed using seroconversion rates of total anti-SARS-CoV-2 S1/S2 IgG, geometric mean titers of anti-S1/S2 IgG (GMT), and frequency of positive neutralizing antibodies (NAb). Physical activity was assessed trough questionnaire (active being defined as $\geq 150 \mathrm{~min} /$ week of moderate-to-vigorous physical activity). Physically active $(n=421)$ and inactive $(n=327)$ ARD patients were similar for most characteristics; however, active patients were significantly younger $(p<0.001)$, had less chronic inflammatory arthritis $(p<0.001)$ and less frequently used biologic agents $(p<0.001)$ than inactive ones. Six months after fullcourse vaccination, being male $(p<0.001)$, use of prednisone $(p<0.01)$ and biologics $(p<0.001)$ were associated with poor immunogenicity, while being physically active was associated with better humoral response $(p<0.01)$. Adjusted point estimates from logistic regression models indicated greater odds of seroconversion rates (OR: 1.5 [95\% Cl: 1.1 to 2.1$]$ ) and NAb positivity (OR: 1.5 [95\% Cl: 1.0 to 2.1]) in physically active patients and approximately $43 \%$ greater $\mathrm{GMT}(42.8 \%$ [ $95 \% \mathrm{Cl}: 11.9$ to 82.2$])$ than inactive ones. In conclusion, among immunocompromised patients, being physically active was associated with an increment in antibody persistence through 6 months after a full-course of an inactivated SARS-CoV-2 vaccine.

\section{Introduction}

Vaccine-induced antibody titers and effectiveness against symptomatic coronavirus infection disease 2019 (COVID-19) have been shown to wane over time. Neutralizing antibodies (NAb) against the B1.351 (Beta) variant were reduced considerably by 6 months among individuals who received the second dose of mRNA-1273 (Moderna's mRNA vaccine). ${ }^{1}$ In addition, humoral response in general population was substantially decreased 6 months after receipt of the second dose of BNT162b2 (Pfizer-BioNTech's mRNA vaccine), especially among men, persons $\geq 65$ years of age, and persons with immunosuppression, with a parallel increase in cases and hospitalization reported in Israel. ${ }^{2,3}$ CoronaVac (Sinovac's inactivated vaccine) is a World Health Organization (WHO) approved vaccine for emergency use that has been shown to be effective in preventing severe cases of COVID- $19^{4}$ and is increasing the global supply through COVAX. ${ }^{5}$ Individuals who received a two-dose schedule of CoronaVac also exhibited a decline in NAb seropositivity by 6 months after full course of vaccination in the general population. ${ }^{6}$ Of note, the same pattern of immunity waning was observed for autoimmune rheumatic disease (ARD) patients vaccinated with CoronaVac, without a concomitant increase in cases or hospitalization, despite the Delta variant spread. ${ }^{7}$ 
In a global scenario with shortage and inequity of vaccines, and heterogeneous responses to vaccination, it is key to gathering knowledge on potential risk factors associated with poor persistence of immunity in order to develop strategies to enhance immunogenicity durability, as well as to prioritize individuals to receive a booster dose. In this regard, there is evidence suggesting that physical activity may act as an adjuvant to vaccines. ${ }^{8,9}$ Among immunocompromised patients vaccinated with two doses of CoronaVac, those who were physically active exhibited higher titers and seroconversion rates than their inactive counterparts. ${ }^{10}$ Whether active individuals show a greater persistence of antibodies than inactive ones remain unclear.

This study aimed to investigate the association between physical activity and persistent humoral immune response 6 months after a two-dose schedule of CoronaVac in patients with ARD.

\section{Methods}

This was a prospective cohort study within an open-label, single-arm, phase 4 vaccination trial (clinicaltrials.gov \#NCT04754698), conducted at a tertiary referral hospital in Sao Paulo, Brazil. The protocol was approved by the institutional ethics committee. Written informed consent was obtained before participants' enrollment.

ARD patients aged $\geq 18$ years and diagnosed with rheumatoid arthritis, systemic lupus erythematosus, axial spondyloarthritis, psoriatic arthritis, primary vasculitis, primary Sjögren's syndrome, systemic sclerosis, idiopathic inflammatory myopathies, and primary antiphospholipid syndrome were eligible. Detailed exclusion and inclusion criteria were described elsewhere. ${ }^{11}$

Patients underwent a two-dose schedule of CoronaVac (Sinovac Life Sciences, Beijing, China, batch \#20200412). ${ }^{11}$ The persistent immunogenicity 6 months after the full-course vaccination was assessed using seroconversion rates of total anti-SARS-CoV-2 S1/S2 IgG (considering values $>15.0 \mathrm{UA} / \mathrm{mL}$ ), geometric mean titers of anti-S1/S2 IgG (GMT), and frequency of positive NAb (inhibition $\geq 30 \%$ ). GMT and NAb assays are thoroughly described elsewhere. ${ }^{10,11}$

Using a telephone-based survey, typical levels of physical activity prior to vaccination were assessed in four domains: leisure time, household activities, work, and commuting. Participants were classified as physically active or inactive according to WHO Guidelines (i.e., physical inactivity defined as $<150$ $\mathrm{min} /$ week of moderate-to-vigorous intensity aerobic activity). ${ }^{12}$

Unadjusted analyses comparing active vs. inactive patients were performed using $\chi^{2}$ test for categorical variables and the Kruskal-Wallis test for continuous variables. Model-based analyses were performed controlling for age ( $<60$ or $\geq 60$ years), sex, and body mass index (BMI) $\left(<25 \mathrm{~kg} / \mathrm{m}^{2} ; 25-30 \mathrm{~kg} / \mathrm{m}^{2} ;>30\right.$ $\left.\mathrm{kg} / \mathrm{m}^{2}\right)$, use of prednisone, immunosuppressants and biologics. Immunogenicity data and physical activity status were added as fixed effects and we conducted logistic regression to estimate odds ratios (ORs) and $95 \% \mathrm{Cls}$ with binary data obtained for rates of IgG seroconversion and NAb positivity. Also, we 
conducted linear regressions for log transformed IgG. Linear regression coefficients and $95 \% \mathrm{Cls}$ for $\log$ transformed dependent variables were back transformed and presented as percent changes. Analyses were conducted using R-statistical environment (R-4.1.0 for Windows).

\section{Results}

A total of 748 ARD patients were analyzed (Table 1 presents baseline characteristics). Physically active $(n=421)$ and inactive $(n=327)$ ARD patients were similar for most characteristics; however, active patients were significantly younger $(p<0.001)$ had a lower frequency of chronic inflammatory arthritis $(p<0.001)$, and less frequently used biologic $(p<0.001)$ than inactive ones (Table 1$)$. 
Table 1

Baseline characteristics of patients with autoimmune rheumatic diseases (ARD) according to physical activity status.

\begin{tabular}{|c|c|c|c|}
\hline & $\begin{array}{l}\text { Active ARD } \\
(n=421)\end{array}$ & $\begin{array}{l}\text { Inactive ARD } \\
(n=327)\end{array}$ & $\begin{array}{l}p- \\
\text { value }\end{array}$ \\
\hline Age, years & $47.0[39.0-59.0]$ & $56.0[45.0-65.0]$ & $<0.001$ \\
\hline Sex, female & $322(76.5)$ & $248(75.8)$ & 0.838 \\
\hline Weight, $k g$ & $71.2[61.3-82.0]$ & $72.0[60.0-83.6]$ & 0.870 \\
\hline Height, $\mathrm{cm}$ & $\begin{array}{l}160.0[155.0- \\
166.0]\end{array}$ & $\begin{array}{l}160.0[154.0- \\
167.0]\end{array}$ & 0.268 \\
\hline $\mathrm{BMI}, \mathrm{kg} / \mathrm{m}^{2}$ & $27.5[24.0-30.9]$ & $27.5[24.2-31.6]$ & \\
\hline Overweight/obese & $267(63.6)$ & 209 (63.9) & 0.923 \\
\hline Caucasian race & $218(51.8)$ & $187(57.2)$ & 0.141 \\
\hline Smoking & $34(8.1)$ & $32(9.8)$ & 0.413 \\
\hline \multicolumn{4}{|l|}{ Comorbidities } \\
\hline Systemic arterial hypertension & $176(41.8)$ & $151(46.2)$ & 0.232 \\
\hline Diabetes mellitus & $40(9.5)$ & $45(13.8)$ & 0.069 \\
\hline Dyslipidemia & $114(27.1)$ & $99(30.3)$ & 0.337 \\
\hline Cardiomyopathy & $27(6.4)$ & $19(5.8)$ & 0.734 \\
\hline Chronic renal disease & $14(3.3)$ & $23(7.0)$ & 0.020 \\
\hline Chronic obstructive pulmonary disease & $3(0.7)$ & $12(3.7)$ & 0.004 \\
\hline Asthma & $17(4.0)$ & $12(3.7)$ & 0.796 \\
\hline Interstitial lung disease & $21(5.0)$ & $39(11.9)$ & 0.001 \\
\hline Pulmonary hypertension & $2(0.5)$ & $6(1.8)$ & 0.073 \\
\hline Hematologic disease & $1(0.2)$ & $1(0.3)$ & 0.858 \\
\hline Hepatic disease & $11(2.6)$ & $20(6.1)$ & 0.017 \\
\hline Cancer & $4(1.0)$ & $4(1.2)$ & 0.719 \\
\hline Stroke & $12(2.9)$ & $11(3.4)$ & 0.687 \\
\hline Tuberculosis & $0(0)$ & $2(0.6)$ & 0.108 \\
\hline \multicolumn{4}{|l|}{ ARD } \\
\hline Chronic inflammatory arthritis (RA, axSpA, PsA) & $185(43.9)$ & $225(69.1)$ & $<0.001$ \\
\hline
\end{tabular}




\begin{tabular}{|c|c|c|c|}
\hline & $\begin{array}{l}\text { Active ARD } \\
(n=421)\end{array}$ & $\begin{array}{l}\text { Inactive ARD } \\
(n=327)\end{array}$ & $\begin{array}{l}p- \\
\text { value }\end{array}$ \\
\hline $\begin{array}{l}\text { Other ARD (SLE, primary vasculitis, SSc, pSSj, } \\
\text { IIM, PAPS) }\end{array}$ & $236(56.1)$ & $102(31.2)$ & $<0.001$ \\
\hline \multicolumn{4}{|l|}{ Current therapy } \\
\hline Prednisone & $155(36.8)$ & $138(42.3)$ & 0.126 \\
\hline Biologic & $145(34.4)$ & $138(42.2)$ & 0.030 \\
\hline Immunosuppressants & $263(62.5)$ & $216(66.1)$ & 0.311 \\
\hline Total physical activity, min per week & $\begin{array}{l}400.0[252.0- \\
720.0]\end{array}$ & $0.0[0.0-75.0]$ & $<0.001$ \\
\hline \multicolumn{4}{|c|}{$\begin{array}{l}\text { Data are presented as median [interquartile range] and } n(\%) \text {. ARD, autoimmune rheumatic disease; } \\
\text { BMI, body mass index; RA, rheumatoid arthritis; axSpA, axial spondyloarthritis; PsA, psoriatic arthritis; } \\
\text { SLE, systemic lupus erythematosus; SSC, systemic sclerosis; pSSj, primary Sjögren syndrome; IIM, } \\
\text { idiopathic inflammatory myopathies; PAPS, primary antiphospholipid syndrome. Biologics include } \\
\text { TNF inhibitor, abatacept, tocilizumab, belimumab, secukinumab, rituximab, ustekinumab. } \\
\text { Immunosuppressants include methotrexate, leflunomide, mycophenolate mofetil, azathioprine, } \\
\text { tofacitinib, cyclophosphamide, tacrolimus and cyclosporine. Total physical activity refers only to time } \\
\text { spent in moderate-to-vigorous intensity activities. }\end{array}$} \\
\hline \multicolumn{4}{|l|}{ Note: Missing data for weight and BMI $(n=1)$. } \\
\hline
\end{tabular}

Figure 1 presents immunogenicity data for active vs. inactive ARD patients. Six months after vaccination, both seroconversion rates of total anti-SARS-CoV-2 S1/S2 IgG (53.1 vs. 40.7\%; $p=0.001)$ and frequency of positive NAb (31.2 vs. $22.0 \% ; p=0.007$ ) were significantly greater in ARD active vs. inactive patients. No differences were found in GMT between the two groups (both $p>0.05$ ).

Figure 2 presents the regression models controlling for covariates. Six months after the full course of vaccination, being male $(p<0.001)$ and use of prednisone $(p<0.01)$ and biologics $(p<0.001)$ were associated with poor immunogenicity, while being physically active was associated with better immunogenicity $(p<0.01)$.

Adjusted point estimates from logistic regression models indicated greater odds of seroconversion rates (OR: 1.5 [95\% Cl: 1.1 to 2.1$]$ ) and NAb positivity (OR: 1.5 [95\%Cl: 1.0 to 2.1$]$ ), and approximately $43 \%$ greater GMT (42.8\% [95\% Cl: 11.9 to 82.2$]$ ) in physically active patients vs. inactive ones.

\section{Discussion}

This study shows that a physically active lifestyle associates with greater immunogenicity 6 months after a two-dose scheme of an inactivated SARS-CoV-2 vaccine among patients with dysfunctional immune system. 
Immunocompromised individuals, such as those with ARD, may experience lower responses to different types of COVID-19 vaccines. ${ }^{11,13}$ Booster doses have been considered for these individuals, but the shortage and inequitable distribution of vaccines across the globe may delay the delivery of extra doses for vulnerable populations. This might be a serious public health issue for many of the over 40 countries administering CoronaVac, particularly those with low and lower middle-income economies.

In this scenario, the identification of predictors of vaccine responses has major implications from clinical and public health perspectives. Recently, we showed that the ARD patients who were physically active exhibited greater seroconversion rates (OR: 1.4 [95\% Cl: 1.1-2.0]) and GMT (32\% [95\% Cl: 8.8-60) vs. their inactive counterparts 6 weeks after the full course of vaccination. ${ }^{10}$ Now, we extended this notion by showing that being physically active is also associated with greater persistent immune response 6 months after vaccination, evidenced by higher rates of seroconversion and neutralizing antibodies. Considering that the prevalence of seropositivity in response to CoronaVac decreased to $17 \%$ following 6 months in general population ${ }^{14}$ and $23.8 \%$ in immunocompromised patients, ${ }^{7}$ the $50 \%$ greater odds of IgG and NAb positivity rates observed herein in active vs. inactive ARD patients appears to be clinically meaningful. The associations observed also suggest that physical activity status may be more influential on antibody persistence than classical factors related to vaccine immunogenicity, such as older age and use of immunosuppressants.

Collectively, our studies conducted within this phase- 4 trial suggest that the significant role of physical activity may not only enhance the humoral immunity to COVID-19 vaccination. ${ }^{10}$, but also sustain its effects over time. This adds to numerous health benefits of being physically active, which includes prevention of several chronic diseases and protection against severe cases of COVID-19. In light of this, global strategies and public health policies focused on tackling physical inactivity become even more relevant and urgent, with special emphasis to individuals with dysfunctional immune system.

The main limitations of this study include its observational design, the lack of estimates of vaccine effectiveness and cell-mediated immune markers and the assessment of physical activity using a subjective tool. Finally, although this study used an inactivated vaccine, one may speculate that our results may be generalizable to other vaccine platforms that yield greater immunogenicity but that at the same time show a more pronounced 6-month decay in immunocompromised patients than Coronovac $2,7,11,13$, suggesting a greater room for improvement with the other platforms.

In conclusion, among immunocompromised ARD patients, being physically activity was associated with greater antibody persistence through 6 months after a full-course of an inactivated SARS-CoV-2 vaccine.

\section{Declarations}

\section{ACKNOWLEDGMENTS}


The authors are thankful to Marta H. Lopes, Tatiana Pedrosa, Antonio José Rodrigues Pereira, Solange Fusco, Priscila Tagliaferro Rojo, and Central Laboratory Division of the Clinical Hospital of the School of Medicine (University of Sao Paulo) for the technical assistance.

\section{AUTHOR CONTRIBUTIONS}

N.E.A., S.G.P., A.C.M.R., C.G.S.S., E.F.N.Y, C.A.S. and E.B., conceived and designed the study, and supervised clinical data management. B.G., I.R.L., R.P.S., A.J.P., B.C.M., F.I.S., S.M.S., N.E.A., S.G.P., A.C.M.R., C.G.S.S., E.F.N.Y, C.A.S., H.R. and E.B participated in data collection. B.G., I.R.L., A.J.P., P.S., P.C.H. and H.R. analyzed and interpreted the data. B.G., H.R., and E.B. wrote the manuscript with inputs from all authors. All authors read and approved the final version.

\section{COMPETING INTEREST STATEMENT}

None declared.

\section{FUNDING}

This trial is sponsored by grants from Fundação de Amparo à Pesquisa do Estado de São Paulo (FAPESP) (\#2015/03756-4 to NEA, SGP, CAS and EB; \#2015/26937-4 to AJP; \#2017/13552-2 to BG; \#2020/04877-8 to IRL; \#2017/23688-9 to RPS; \#2019/14820-6 to BCM; \#2019/14819-8 to FIS; \#2019/15231-4 to SMS), Conselho Nacional de Desenvolvimento Científico e Tecnológico (CNPq) (\#305242/2019-9 to EB, \#304984/2020-5 to CAS) and B3 - Bolsa de Valores do Brasil. Instituto Butantan supplied the study's product and had no other role in the trial.

\section{Data availability statement}

All background and clinical information for ARD patients in this study are available from the corresponding author on reasonable request.

\section{References}

1. Pegu A, O'Connell SE, Schmidt SD, et al. Durability of mRNA-1273 vaccine-induced antibodies against SARS-CoV-2 variants. Science. 2021;373(6561):1372-1377. doi:10.1126/science.abj4176

2. Levin EG, Lustig Y, Cohen C, et al. Waning Immune Humoral Response to BNT162b2 Covid-19 Vaccine over 6 Months. N Engl J Med. 2021;385(24):e84. doi:10.1056/NEJMoa2114583

3. Barda N, Dagan N, Cohen C, et al. Effectiveness of a third dose of the BNT162b2 mRNA COVID-19 vaccine for preventing severe outcomes in Israel: an observational study. Lancet (London, England). 2021;398(10316):2093-2100. doi:10.1016/S0140-6736(21)02249-2

4. Jara A, Undurraga EA, González C, et al. Effectiveness of an Inactivated SARS-CoV-2 Vaccine in Chile. N Engl J Med. 2021;385(10):875-884. doi:10.1056/NEJMoa2107715 
5. Gavithe Vaccine Alliance. COVAX Global Supply Forecast. Accessed December 16, 2021. https://www.gavi.org/sites/default/files/covid/covax/COVAX-Supply-Forecast.pdf

6. Zeng G, Wu Q, Pan H, et al. Immunogenicity and safety of a third dose of CoronaVac, and immune persistence of a two-dose schedule, in healthy adults: interim results from two single-centre, doubleblind, randomised, placebo-controlled phase 2 clinical trials. Lancet Infect Dis. Published online December 2021. doi:10.1016/S1473-3099(21)00681-2

7. Bonfa E, Silva C, Medeiros-Ribeiro A, et al. Anti-SARS-CoV-2 immunogenicity decay and incident cases six months after Sinovac-CoronaVac inactivated vaccine in autoimmune rheumatic diseases patients: phase 4 prospective trial. 23 November 2021, PREPRINT (Version 1) available at Research Square [https. Published online 2021. doi:10.21203/rs.3.rs-1054476/v1

8. Simpson RJ, Kunz H, Agha N, Graff R. Exercise and the Regulation of Immune Functions. Prog Mol Biol Trans/ Sci. 2015;135:355-380. doi:10.1016/bs.pmbts.2015.08.001

9. Chastin SFM, Abaraogu U, Bourgois JG, et al. Effects of Regular Physical Activity on the Immune System, Vaccination and Risk of Community-Acquired Infectious Disease in the General Population: Systematic Review and Meta-Analysis. Sports Med. Published online April 2021:1-14. doi:10.1007/s40279-021-01466-1

10. Gualano B, Silva R, Pinto A, et al. Physical activity associates with enhanced immunogenicity of an inactivated virus vaccine against SARS-CoV-2 in patients with autoimmune rheumatic diseases. Pre Print (Research Square). Published online 2021. doi:10.21203/rs.3.rs-782398/v1

11. Medeiros-Ribeiro AC, Aikawa NE, Saad CGS, et al. Immunogenicity and safety of the CoronaVac inactivated vaccine in patients with autoimmune rheumatic diseases: a phase 4 trial. Nat Med. Published online July 2021. doi:10.1038/s41591-021-01469-5

12. Bull FC, Al-Ansari SS, Biddle S, et al. World Health Organization 2020 guidelines on physical activity and sedentary behaviour. Br J Sports Med. 2020;54(24):1451-1462. doi:10.1136/bjsports-2020102955

13. Furer V, Eviatar T, Zisman D, et al. Immunogenicity and safety of the BNT162b2 mRNA COVID-19 vaccine in adult patients with autoimmune inflammatory rheumatic diseases and in the general population: a multicentre study. Ann Rheum Dis. 2021;80(10):1330-1338.

doi:10.1136/annrheumdis-2021-220647

14. World Health Organization (WHO). Rcommendation for an Emergency Use Listing of COVID-19 Vaccine (Vero Cell), Inactivated Submitted by Sinovac. (June 28, 2021). https://extranet.who.int/pqweb/sites/default/files/documents/SINOVAC_TAG_PEG_REPORT_EULFinal28june2021.pdf

\section{Figures}



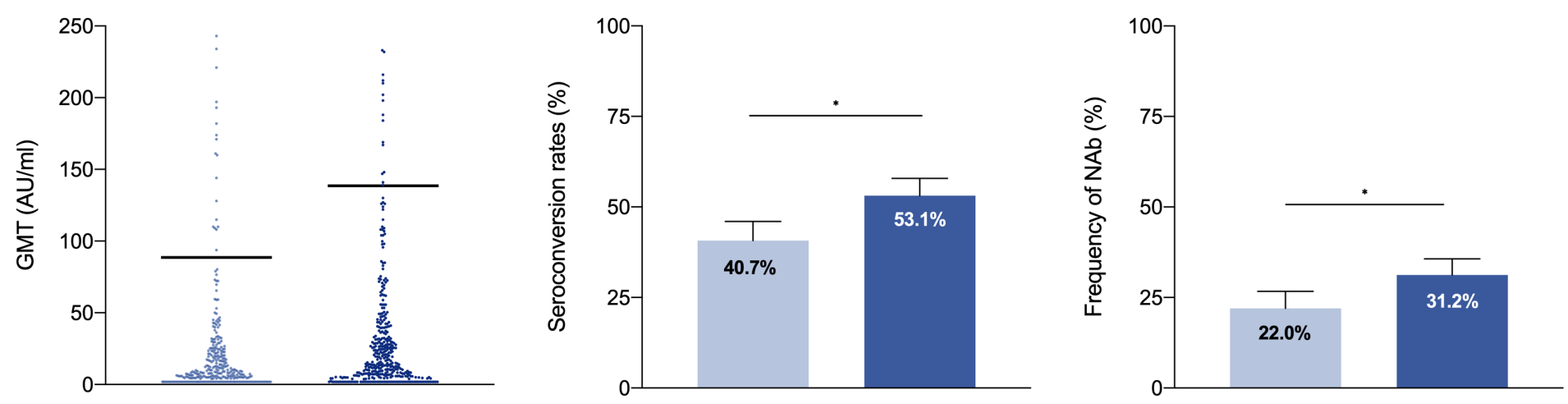

\section{Figure 1}

Unadjusted analysis for immunogenicity data in autoimmune rheumatic diseases patients (ARD) six months after full vaccination with CoronaVac.

${ }^{\star} P<$.05. Seroconversion was defined as a positive serology (IgG titer $\geq 15 \mathrm{AU} / \mathrm{ml}$ ) for anti-SARS-CoV-2 $\mathrm{S} 1 / \mathrm{S} 2$ IgG antibodies after vaccination (Indirect ELISA, LIAISON® SARS-CoV-2 S1/S2 IgG, DiaSorin, Italy). Positivity for NAb was defined as a neutralizing activity $\geq 30 \%$ (cPass sVNT Kit, GenScript, Piscataway, USA). Data are expressed as individual data, median and interquartile range for GMT and percentages and $95 \%$ confidence intervals (Cls) for frequency of seroconversion rates of total anti-SARS-Cov-2 S1/S2 IgG (SC) and neutralizing antibodies (NAb) positivity. Missing data for IgG $(n=3)$ and NAb $(n=43)$. 
Seroconversion rates (\%) OR $[95 \% \mathrm{Cl}]$
GMT (AU/ml)

$\%$ change $[95 \% \mathrm{Cl}]$
Frequency of NAb (\%)

$\mathrm{OR}[95 \% \mathrm{Cl}]$

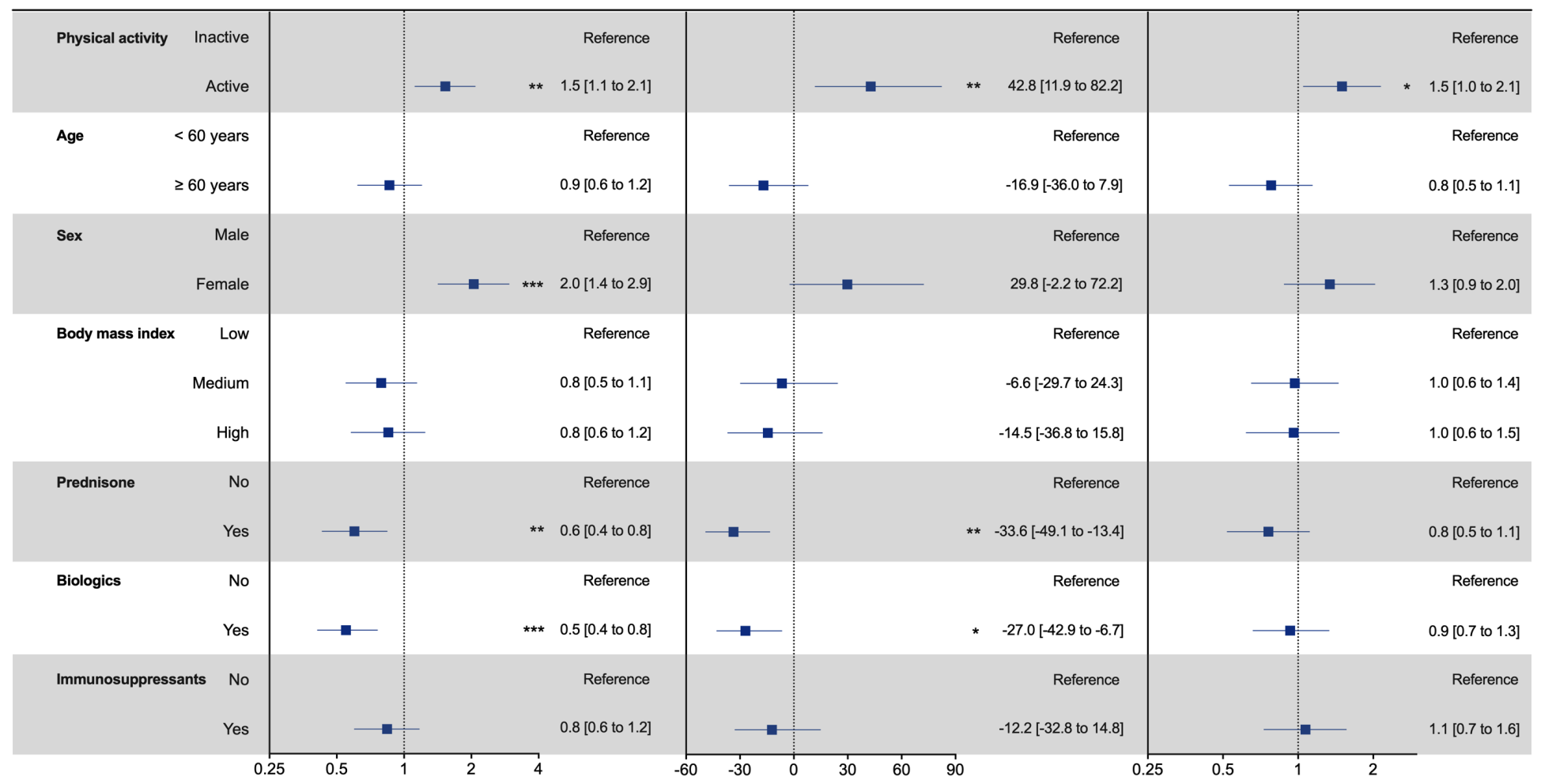

Figure 2

Adjusted risk factors for immunogenicity data in autoimmune rheumatic diseases (ARD) patients six months after full vaccination with CoronaVac.

Logistic regression to estimate odds ratios (ORs) and 95\% confidence intervals (Cls) with binary data obtained for frequency of seroconversion rates of total anti-SARS-Cov-2 S1/S2 IgG (SC) and neutralizing antibodies (NAb) positivity. Linear regression was used for natural log transformed GMT. Data expressed as percent change [95\% $\mathrm{Cl}]$ ). Adjusted for age, sex, $\mathrm{BMI}$, use of prednisone, immunosuppressants and biologics. ${ }^{*} P<.05,{ }^{*} P<.01, * \star \star P<.001$. Seroconversion was defined as a positive serology (IgG titer $\geq 15$ $\mathrm{AU} / \mathrm{ml}$ ) for anti-SARS-CoV-2 S1/S2 IgG antibodies after vaccination (Indirect ELISA, LIAISON® SARS-CoV$2 \mathrm{~S} 1 / \mathrm{S} 2$ IgG, DiaSorin, Italy). Positivity for NAb was defined as a neutralizing activity $\geq 30 \%$ (cPass sVNT Kit, GenScript, Piscataway, USA). Missing data for IgG $(n=3)$ and NAb $(n=43)$. 